\title{
Frontières
}

\section{Ces pédopsychiatres que T. et Y. n’ont pas consultés}

\section{Denis Lafortune, Nadine Deslauriers-Varin et Rachel Bouchard}

Volume 16, numéro 1, automne 2003

Remède ou poison?

URI : https://id.erudit.org/iderudit/1073765ar

DOI : https://doi.org/10.7202/1073765ar

Aller au sommaire du numéro

Éditeur(s)

Université du Québec à Montréal

ISSN

1180-3479 (imprimé)

1916-0976 (numérique)

Découvrir la revue

Citer cet article

Lafortune, D., Deslauriers-Varin, N. \& Bouchard, R. (2003). Ces pédopsychiatres que T. et Y. n'ont pas consultés. Frontières, 16(1), 78-84.

https://doi.org/10.7202/1073765ar

\section{Résumé de l'article}

Ce travail s'inscrit dans le cadre d'un programme de recherche plus large, traitant du recours à la psychopharmacothérapie dans la prise en charge des jeunes placés en centres jeunesse. Il s'agit ici de comprendre plus spécifiquement comment l'intervention des éducateurs en matière de risques suicidaires a été influencée, voire même transformée par deux suicides d'adolescents survenus en 1994 et 1997, suivis d'une série de rapports d'enquêtes, d'autopsies psychologiques et de protocoles d'intervention. 


\section{Résumé}

Ce travail s'inscrit dans le cadre d'un programme de recherche plus large, traitant du recours à la psychopharmacothérapie dans la prise en charge des jeunes placés en centres jeunesse. II s'agit ici de comprendre plus spécifiquement comment l'intervention des éducateurs en matière de risques suicidaires a été influencée, voire même transformée par deux suicides d'adolescents survenus en 1994 et 1997, suivis d'une série de rapports d'enquêtes, d'autopsies psychologiques et de protocoles d'intervention.

Mots clés : suicide - jeunes - éducateurs thérapie.

\begin{abstract}
This work is part of a larger research program dealing with recourse to psychopharmacotherapy in handling youths placed in youth centers. Specifically, this paper attempts to understand how the intervention of educators, with respect to the management of suicide risk, has been influenced, if not transformed, by two adolescent suicides that occurred in 1994 and 1997, which were followed by a series of investigations, psychological autopsies and intervention protocols.
\end{abstract}

Keywords: suicide - youth - educators therapy.

\section{CES PÉDOPSYCHIATRES QUE T. ET Y. N'ONT PAS CONSULTÉS}

Denis Lafortune, Ph.D.,

professeur, École de criminologie, Université de Montréal.

Nadine Deslauriers-Varin, École de criminologie, Université de Montréal.

Rachel Bouchard, M.Sc., École de criminologie, Université de Montréal.

\section{LE CONTEXTE DEPUIS NEUF ANS}

En 1994, T., âgé de 14 ans, fils aîné de deux médecins québécois, les Dr A. et B., met fin à ses jours.

Dans les mois qui suivent le drame, ses parents entreprennent une longue campagne "de sensibilisation », une croisade en fait. Ils souhaitent que les divers intervenants du réseau de la santé et des services sociaux sachent "mieux diagnostiquer la dépression chez les adolescents », un phénomène qu'ils disent méconnu.

Trois ans plus tard, en décembre 1997, survient un autre triste événement: Y., un jeune homme, est retrouvé pendu dans sa chambre au Centre jeunesse de Laval. Bien que le couple A. et B. n'ait aucun lien de parenté avec ce garçon, il formule une plainte aux autorités. En résumé, les deux médecins reprochent au Centre jeunesse de ne pas avoir demandé l'évaluation pédopsychiatrique qui, à leurs yeux, aurait été nécessaire pour prévenir ce suicide. Et ils obtiennent que le Bureau du coroner fasse enquête sur le décès de Y.
En novembre 1998, le coroner Anne M. David rend public son rapport (Bureau du Coroner, 1998). Dans un premier temps, l'auteure trace un portrait de l'adolescent. Par la suite, elle se penche sur les cinq mois de séjour du jeune homme au centre et analyse la prise en charge «type » d'un jeune dans un tel établissement. Finalement, le coroner rend publiques les impressions diagnostiques d'un pédopsychiatre de l'Hôpital Sainte-Justine concernant l'adolescent et l'évaluation des interventions pratiquées auprès de celui-ci. En fin de texte, diverses recommandations sont faites au ministère de la Santé et des Services Sociaux, notamment: $a$ ) de s'assurer que les travailleurs sociaux peuvent recourir à un pédopsychiatre lorsqu'un jeune est pris en charge par la protection de la jeunesse et $b$ ) de former un comité multidisciplinaire composé de représentants des centres jeunesse, du Collège des médecins du Québec et de l'Association des hôpitaux du Québec. Le comité proposé aurait pour mandat de mettre sur pied un mécanisme de liaison entre les intervenants des centres jeunesse et ceux des milieux médicaux. Il est enfin souhaité que l'on confie à une équipe de pédopsychiatres la tâche de procéder à l'étude systématique des cas de suicide survenus dans les centres de réadaptation au cours des cinq dernières années.

Or, en janvier 1998, quelques mois avant la parution du rapport du Coroner, un comité semblable avait déjà vu le jour. 
Formé de représentants de l'Association des centres jeunesse du Québec, du Collège des médecins du Québec et du Protecteur du citoyen, le "Comité sur le suicide des jeunes » s'est donné pour mandat de proposer des «moyens concrets [permettant] de réaliser l'arrimage des ressources des milieux médicaux et sociaux en précisant le rôle des différentes ressources et les liens qu'elles doivent entretenir entre elles. »

Dans ses travaux, le comité mène sa propre recherche, avec l'accord de la Commission d'accès à l'information, sur les suicides de jeunes en centres jeunesse. Il constate qu'en 1995 et 1996, 57 des 177 jeunes québécois suicidés (soit 32 \%) étaient connus des centres jeunesse. Quarante-cinq de ces 57 jeunes étaient effectivement suivis par les centres jeunesse au moment de leur geste fatal. Les douze autres avaient déjà reçu des services de ces établissements au cours des trois dernières années. Par ailleurs, 79 \% des jeunes suicidés (140/177) avaient vu un médecin dans l'année précédant leur décès et $24 \%$ avaient même vu un médecin durant le mois précédant leur suicide. En reculant la fenêtre d'observation jusqu'à cinq ans avant le passage à l'acte, on observait enfin que $44 \%$ de ces jeunes avaient consulté en pédopsychiatrie. Plusieurs jeunes suicidés étaient donc déjà connus d'un centre jeunesse ou, le plus souvent, d'un médecin. Dès lors, comment comprendre l'issue tragique de ces consultations et des suivis amorcés? Il faut, selon les auteurs, l'attribuer à un manque de concertation.

\section{LE RAPPORT : LE SUICIDE CHEZ LES USAGERS DES CENTRES JEUNESSE : IL EST URGENT D'AGIR}

En avril 1999, ce Comité triparti dépose un rapport intitulé Le suicide chez les usagers des centres jeunesse : il est urgent d'agir. Les idées maîtresses en sont: la concertation et la collaboration entre les intervenants, l'accès à des services médicaux de qualité, l'acceptation des spécificités et limites de chacun des groupes professionnels, la nécessité de formations communes, la synergie, la coordination, des ententes de service, des protocoles d'intervention et le partage des responsabilités. De manière générale, les auteurs déplorent les écarts de pensée qui créent, entre les membres des diverses professions médicales et psychosociales, des tensions. Ils écrivent: «trop de professionnels perçoivent les actions des autres professionnels comme autant d'intrusions dans leur champ de compétence spécifique » (section 4, p. 7). À la fin du document, 24 recommandations sont formulées quant au leadership que doit assurer le ministère de la Santé et des Services sociaux, aux ressources médicales spécialisées en santé mentale qu'il faut rendre accessibles (omnipraticiens avec formation additionnelle en psychiatrie et pédopsychiatres), aux actions à mettre en œuvre, aux normes de dépistage, d'intervention, de tenue de dossier et de transfert d'information qu'il faut développer, à l'évaluation de l'efficacité de leurs interventions que devraient faire les centres jeunesse et à la formation spécialisée en matière de suicide qui devrait être donnée à tous les intervenants, du réseau psychosocial ou du réseau médical.

D'autres passages critiquent, à mots à peine couverts, les pratiques en centres jeunesse. Ainsi, les chercheurs disent "avoir observé qu'une proportion importante d'intervenants étaient incapables de se prononcer sur la présence ou l'absence de caractéristiques hautement associées au risque suicidaire chez les jeunes dont ils avaient nouvellement la charge » (section 3 , p. 4). Un peu plus loin, on peut aussi lire que les « psychiatres déplorent chez les intervenants des CJ l'insuffisance des connaissances pour le dépistage des troubles en santé mentale» (section 4, p. 9). Or, cette observation ne s'accompagne d'aucune interrogation réciproque sur le niveau de connaissance des problématiques propres aux centres jeunesse par les pédopsychiatres. Aussi, n'est-on pas surpris d'apprendre que les "psychiatres ne sont pas toujours intéressés à travailler avec les intervenants des CJ » (section 4, p. 9). Quelques lignes plus loin, on rappelle que plusieurs facteurs contribuent au manque de concertation, notamment « le caractère empirique des connaissances dans les disciplines de sciences humaines et la difficulté d'en mesurer les résultats » (section 4, p. 10). La $20^{\mathrm{e}}$ recommandation veut donc que les centres jeunesse procèdent à :

\section{[...] l'évaluation de l'efficacité de}

leurs interventions portant sur d'autres facteurs suicidaires, tels que le comportement antisocial, l'impulsivité, la toxicomanie, les troubles cognitifs, les pertes relationnelles, les conflits interpersonnels et les changements de milieux subis par la clientèle, ainsi que le niveau de stress associé à certaines interventions psychosociales et judiciaires (Section 8, p. 4).

De nouveau, il est frappant qu'aucune réciproque ne soit proposée quant à l'évaluation et à la bonification que les médecins pourraient faire de leurs propres pratiques.

Sur le plan clinique, le Comité propose diverses avenues. En premier lieu, le modèle bio-psycho-social retenu par la psychiatrie depuis une vingtaine d'années est présenté comme un terrain d'entente pouvant fonder les pratiques de tous les groupes d'intervenants. Au fil du texte, le suicide est défini comme le signe d'une psychopathologie, d'un désordre psychique, puis d'une maladie mentale. En deux endroits, on peut lire, sans que les sources bibliographiques ne soient citées, que « chez $85 \%$ des jeunes qui se suicident, on a pu constater la présence de maladies mentales». Il appartiendrait donc aux médecins de diagnostiquer les maladies physiques et mentales des jeunes, de déterminer la gravité de ces maladies et de définir les traitements appropriés. Les auteurs soutiennent que :

Les composantes biologiques de plusieurs psychopathologies ont été identifiées et des traitements pharmacologiques ont été développés. Par ailleurs, des psychothérapies de plus en plus spécifiques reposant sur des approches cognitivo-comportementales ont été également introduites et se révèlent efficaces, surtout lorsque combinées aux pharmacothérapies

(Section 4, p. 6).

En somme, on voudrait voir les médecins et les intervenants de centres jeunesse a) travailler en plus étroite collaboration, b) dans un cadre conceptuel relativement large, mais qui est, de fait, issu de la pédopsychiatrie, $c$ ) en fonction d'une prémisse voulant que chez $85 \%$ des jeunes suicidaires on trouve une maladie mentale et d) en envisageant assez rapidement le recours aux pharmacothérapies et aux thérapies cognitives.

\section{LES RETOMBÉES}

Dans la suite des événements, le Collège des médecins du Québec émet une position sur l'accessibilité des soins psychiatriques à la clientèle adolescente et les centres jeunesse révisent leurs protocoles d'intervention, le ministre délégué à la Jeunesse fait un investissement de 27,5 millions de dollars, un atelier de formation («Le médecin et l'ado qui déprime ») est mis sur pied à l'intention des médecins, tandis que la Fondation des maladies mentales du Québec effectue la tournée de sensibilisation "Solidaires pour la vie » auprès des jeunes des écoles secondaires. Finalement, en 2001, le travail du couple A. et B. est souligné par le Collège des médecins du Québec, qui leur décerne un Grand Prix pour leur contribution à l'amélioration de la médecine (Collège des médecins, 2001).

Durant la période qui suit la remise de ce prix, la mère (Dr A.) rencontre une journaliste et elle l'interpelle: «Quand on vous a parlé du suicide, vous a-t-on déjà dit qu'il pouvait être la conséquence d'une maladie? Pour découvrir si un enfant souffre de dépression et risque de se suicider, il faut 
d'abord être en mesure de se poser cette question. Mais pour cela, il faut détenir cette information!»(Bousquet, 2002, p. 3). La journaliste ajoute dans son texte :

La dépression chez l'adolescent est pourtant reconnue médicalement depuis le début des années 1980. Mais on continue de croire que le suicide est un phénomène social qui relève d'un mal de vivre, au lieu de penser qu'il fait partie de la dépression. Et qu'il en est l'aboutissement si elle n'est pas traitée! [...]. C'est le médecin qui devra poser le diagnostic. [...] Le traitement, quant à lui, présente généralement deux facettes: des médicaments à prendre et une thérapie à suivre, en complément (Bousquet, 2002, p. 4).

Et la mère de $\mathrm{T}$. de conclure que les parents «n'agiront pas de la même façon avec leur enfant malade qu'avec un enfant simplement dissipé. Connaître sa maladie leur permettra de mieux le soutenir, l'encourager et le dorloter» (Bousquet, 2002, p. 4).

\section{L'AMPLEUR DU PHÉNOMÈNE}

Pronovost et Leclerc (1998) ont analysé la situation de 351 jeunes âgés de 11 à 18 ans dans les premières semaines de leur admission en centre jeunesse au Québec, entre octobre 1997 et juin 1998. Ils établissent qu'au moment de la collecte de données, $33 \%$ de ces jeunes avaient fait une tentative de suicide au cours de leur vie. De ce nombre, la moitié des tentatives était attribuée par les jeunes à des problèmes familiaux, tandis que $46 \%$ des gestes étaient associés à des volets du placement en centre jeunesse, soit: la non-acceptation du placement, l'attente du placement, la modification de celui-ci ou sa prolongation. Une autre étude menée par Chagnon (1998, dans Comité sur le suicide des jeunes, 1999) auprès de 160 jeunes de 14 à 17 ans admis en internat dans deux établissements des centres jeunesse de Montréal entre avril 1995 et mai 1996 présente des résultats comparables: $39 \%$ des répondants indiquent avoir fait une tentative de suicide au cours des trois dernières années. Du point de vue de l'intervenant, la question de la condition dépressive et du risque suicidaire se pose donc pour le tiers des jeunes accompagnés.

\section{PERCEPTIONS DES ÉDUCATEURS EN 2001-2002}

Nous avons introduit cette communication par un contexte historique, plutôt que par un contexte théorique. Il en est ainsi parce que la question de recherche qui nous préoccupe est celle-ci : comment les éducateurs perçoivent-ils leur rôle en cette époque d'enquête du coroner, de codes et de procédures? Les entretiens analysés dans la partie qui suit s'inscrivent dans le cadre d'une recherche plus large, traitant du recours aux médicaments psychotropes dans les centres jeunesse (Bouchard, 2002; Lafortune et Laurier, 2003 ; Lafortune, Laurier et Gagnon, 2003). Pour saisir le point de vue et les perceptions qu'entretiennent les éducateurs en centres jeunesse à l'égard de la psychopharmacothérapie, dix-neuf entretiens semi-directifs ont été menés. Les répondants travaillaient dans une ressource de rééducation des centres jeunesse de Laval ou de Montréal, qu'il s'agisse d'un foyer de groupe, d'une unité régulière ou d'une unité d'encadrement intensif. Ils devaient avoir eu la responsabilité d'au moins un adolescent sous médication psychotrope. D'un âge variant entre 27 et 46 ans, ils avaient pour la plupart (13 / 19) un baccalauréat universitaire, les autres détenant une maîtrise (2) ou un diplôme d'études collégiales (4). Le nombre d'années d'expérience se situait entre 3,5 et 24 ans, la moyenne étant de 14 ans. Les horaires de travail de la plupart des interviewés alternaient entre les quarts de jour, de soir et de fins de semaine.

Les entretiens, d'une durée moyenne de 90 minutes, ont été menés autour des questions suivantes : Quelle est la place qu'occupe la psychopharmacologie dans la pratique clinique des éducateurs en centres jeunesse? Quelles perceptions ces éducateurs ont-ils de la pharmacothérapie, de ses indications et de son efficacité ? Dans quels contextes se pose pour eux la question de proposer ou de recommander une médication psychotrope? Quels enjeux cliniques et éthiques le recours aux médicaments psychotropes soulève-t-il ? (Bouchard, 2002). De l'ensemble des 19 entrevues, 16 ont été sélectionnées pour faire l'objet d'une seconde analyse thématique parce qu'il y était explicitement question de risque suicidaire pour les jeunes résidants. Cette analyse secondaire a été faite par deux personnes travaillant indépendamment l'une de l'autre (les deux premiers auteurs), qui ont ensuite discuté entre eux de leur découpage.

\section{LE PROTOCOLE D'INTERVENTION LIANT LES CENTRES JEUNESSE AUX MILIEUX MÉDICAUX}

De manière générale, les éducateurs rencontrés considèrent qu'une part importante des jeunes dont ils s'occupent présente des signes de troubles mentaux. Parmi ceux-ci, hormis l'impulsivité, l'hyperactivité et le déficit d'attention, figurent surtout l'automutilation, les idéations suicidaires et les tentatives de suicide. Pour de tels jeunes, les propos des éducateurs indiquent que la consultation médicale et la pharmacothérapie jouent un rôle de premier plan

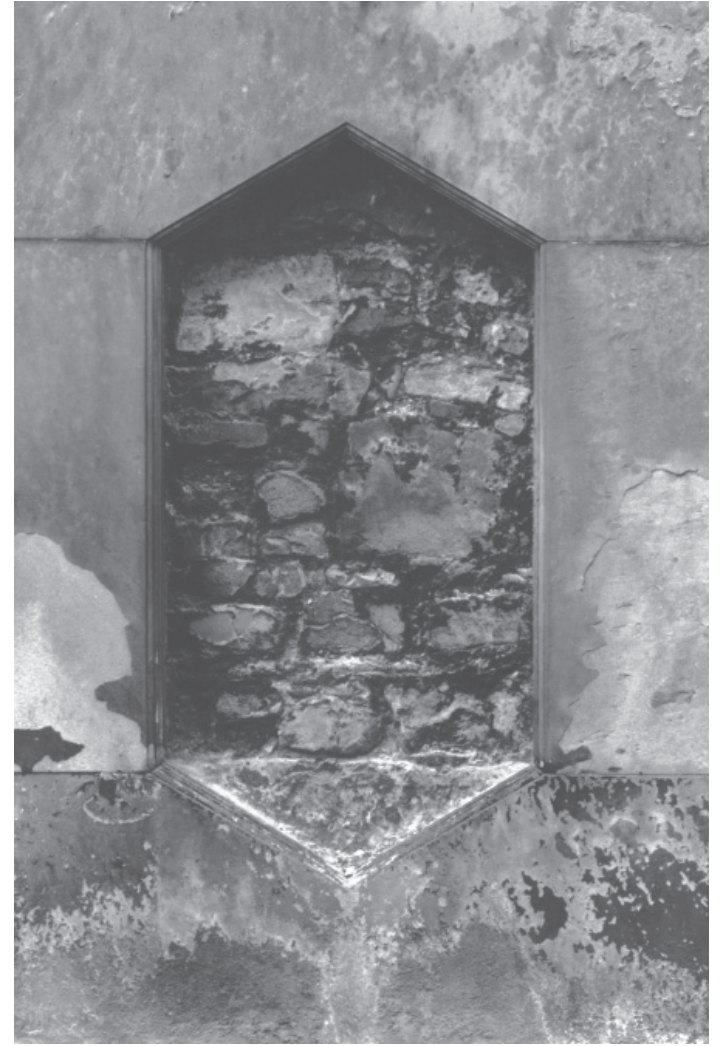

puisque le simple «plan d'intervention » en centre d'accueil ne convient plus. Coincés entre les milieux médicaux et ceux de la réadaptation, ces adolescents font de brefs séjours à l'hôpital, via l'urgence, puis retournent dans leurs unités, une situation qui paraît déconcertante pour les intervenants. Cette variation sur le thème de la «porte tournante » paraît bien illustrer le manque de concertation et de synergie déploré par le Comité sur le suicide des jeunes en 2001.

- Là, ils étaient en pédopsychiatrie parce qu'ils avaient eu une crise, genre tentative de suicide ou mutilation trop grave. Là, ils allaient à l'hôpital. Eux les contenaient physiquement, chimiquement, mécaniquement aussi avec les filets. Ben là, «On ne voit plus rien. Il n'y a plus de comportement. Ce n'est plus un cas pour nous autres. On vous la renvoie».

Un grand nombre d'éducateurs apprécient le protocole d'intervention mis en place par l'Association des centres jeunesse et le Collège des médecins, à la suite des événements du site Cartier, à Laval, protocole qui stipule qu'au moindre doute, un médecin doit évaluer la gravité du risque. Les répondants soulignent qu'il s'agit là d'un partenariat tout à fait rassurant, caractérisé par la complémentarité et la collaboration. Conscients des réactions affectives que provoquent en eux de tels comportements, les praticiens disent faire des efforts pour maintenir une position 


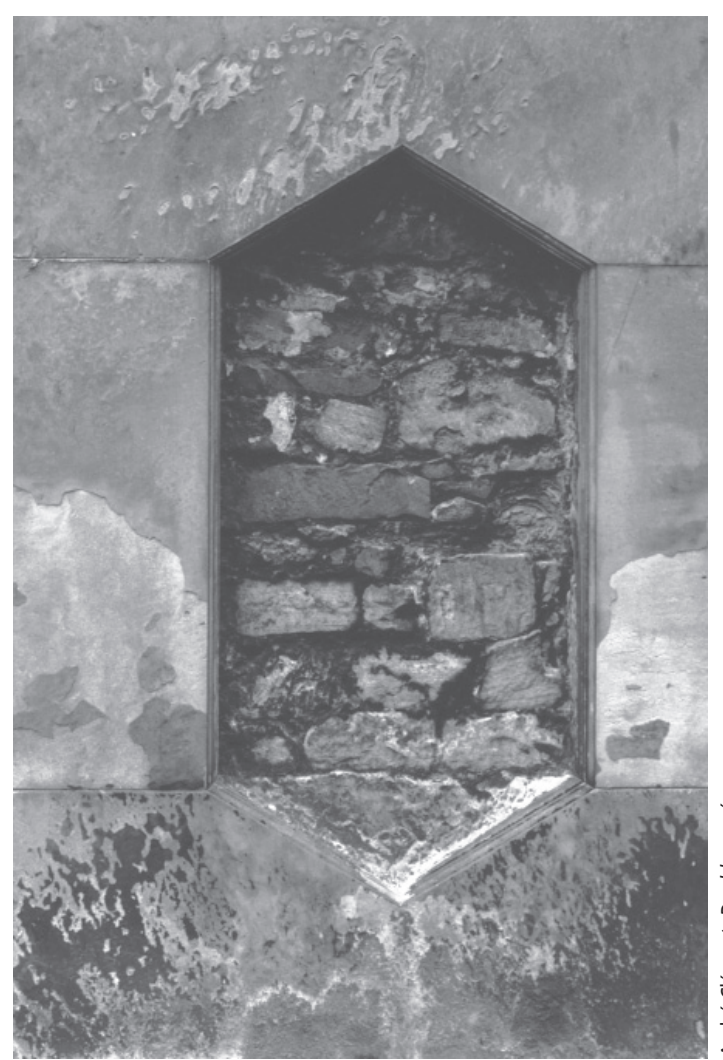

neutre et objective. Ils accumulent alors les observations jugées pertinentes, ils en discutent en équipe, puis ils envisagent d'entreprendre une démarche auprès du médecin.

- Oui, mais nous autres, faut faire la demande avec les éléments qu'on observe sur le plancher avec ces jeunes-là.

- C'est qu'on regarde à tous les niveaux, que ça soit son secteur familial, scolaire, au niveau de sa santé aussi. Donc, la médication fait partie de sa santé.

- Maintenant, c'est facile. Si l'adolescente fait des verbalisations suicidaires, c'est sûr que tu vas consulter à

l'urgence. Ça, c'est suite à des suicides qu'il y a déjà eus au centre Cartier. [...] Donc, là-dedans, on est protégé par cette loi-là. Ce qui fait que maintenant c'est plus facile. Mais là, je remonte au temps où je te parle. C'étaient nous autres qui décidaient si c'était dangereux ou pas.

\section{LE PROTOCOLE D'INTERVENTION, UNE « ASSURANCE RESPONSABILITÉ ॥ ?}

Le dernier extrait suggère que, pour quelques intervenants (six), la mise en place du protocole d'intervention a été ressentie comme un soutien, voire comme une protection en cas de décisions difficiles, de manifestations devant lesquelles ils se sentent insuffisamment formés pour assumer une prise en charge. D'autres éducateurs (quatre) estiment par contre que le personnel des centres jeunesse est devenu extrêmement prudent, voire précautionneux face au suicide. Ils évoquent presque un climat de panique, en relatant la mise en place de ce protocole et la multiplication des consultations d'urgence qui lui a succédé.

- De toute façon, il est arrivé aussi des événements dans la maison qui font qu'ils ne veulent pas perdre ça de vue non plus. Tsé, ils sont vraiment à l'affût de ces affaires-là.

- «Suicide», ça ouvre toutes les portes dans les centres jeunesse.

- Aussitôt qu'un jeune nous parle qu'il a déjà fait une tentative ou qu'il y a déjà pensé ou qu'il se sent un petit peu dépressif, c'est comme s'il s'installe toute une machine, tout un déroulement d'actions, là.

- On a une responsabilité très lourde à ce niveau-là.

Aux yeux de ces interviewés, l'étroite vigilance qui prévaut n'est pas sans occasionner de nouveaux problèmes. Il existe d'abord des risques de manipulation de la part de certains jeunes, qui seraient à la recherche de bénéfices secondaires :

- Il reste qu'on lui a prescrit un médicament suite à l'ensemble de ses menaces de suicide, puis de sa recherche continuelle d'attention, pour avoir tout le temps de l'attention.

Toutefois les répondants considèrent que le principal effet iatrogène du protocole d'intervention réside plutôt dans la crainte insidieuse "d'être blâmés». Les enquêtes $\mathrm{du}$ coroner et autopsies psychologiques paraissent en avoir ébranlé quelques-uns et installé un climat d'insécurité. Soucieux de prendre un minimum de risque, ceux-là réfèrent au milieu médical dans un mouvement qui semble être devenu un réflexe défensif, un désir d'autoprotection, voire un automatisme. En somme, la consultation à l'urgence devient parfois l'unique et seule solution envisagée.

- On a quand même un protocole ici. Verbalisations suicidaires, agirs mutilatoires, puis passages à l'acte suicidaire. Ça, c'est automatique. Là, on ne se questionne pas.

- C'était tout de suite d'aller évaluer le danger suicidaire pour être ben, ben sûrs que professionnellement j'ai tout fait ce que j'avais à faire. On est tombé dans des excès épouvantables, selon moi. Et tout était teinté par «la crainte de... », puis de s'assurer qu'on balise tout, tout, tout comme il faut pour éviter qu'il se passe un suicide.

- Nous, on n'est pas équipés à ce niveau-là.

- Ça fait que pourquoi diminuer une médication si le risque augmenterait peut-être? C'est comme s'ils ne prennent pas de chance, mais vraiment pas de chance, là. On va maintenir ça comme ça. C'est plus safe. C'est plus sécuritaire pour tout le monde. On n'aura pas de problème.

\section{LE RAPPORT AMBIVALENT DES ÉDUCATEURS À L'EXPERTISE MÉDICALE}

Commentant leurs liens avec les milieux médicaux, plusieurs des répondants ont dit en attendre un échange, un éclairage, voire une expertise en matière de psychopathologie et de suicide. Ils espèrent aussi une référence plus rapide aux services ambulatoires et réguliers qui leur paraissent caractérisés par de (très) longues listes d'attente. La relation qu'entretiennent les éducateurs avec les pédopsychiatres semble s'améliorer au fil des années. En effet, les premiers se disent de plus en plus reconnus comme des collaborateurs par des médecins disposés à tenir compte de leurs remarques sur la situation des jeunes résidants. De façon générale, ils jugent pouvoir communiquer avec le médecin par écrit, par téléphone ou encore en accompagnant le jeune lors de l'entretien à l'hôpital.

L'établissement de ces liens de réciprocité ne paraît toutefois pas aller de soi pour tous. En effet, loin d'être un coéquipier ou un partenaire intéressé aux échanges, certains pédopsychiatres sont plutôt décrits comme étant distants, débordés et peu disponibles. En contrepartie, il nous a semblé que certains éducateurs se plaçaient, eux, en position d'attente passive devant le corps médical. Plusieurs de nos interviewés ont fait allusion à des expériences malheureuses de suivis cloisonnés, sans échanges, décevants, leur laissant l'impression que le médecin portait peu d'intérêt à leur propre lecture de la situation.

- En ce moment, c'est les psychiatres qui nous disent que: " la dynamique de l'adolescente est état-limite. O.K., ce n'est pas cristallisé, ce n'est pas officiel. Peut-être qu'après 18 ans elle aura ce diagnostic-là ou pas. Mais c'est sûr que dans la dynamique affective de cette jeune-là, il y a beaucoup de caractéristiques état-limite». C'est la psychiatrie qui nous comprend cette adolescentelà comme ça. Donc, la qualification vient du psychiatre, mais il y a aussi le 
psychologue consultant qui voit la jeune dans cette dynamique-là.

- Bon, on vous envoie tel jeune, tel type de dynamique, tout ça. On vous l'envoie. Puis eux, ils nous renvoient le diagnostic ou l'évaluation du psychiatre.

- Là, on tombe dans le service médical, et eux-autres repartent leur évaluation sans nécessairement tenir compte de qu'est-ce qu'on a fait. C'est vrai que c'est un peu frustrant à ce niveau-là.

\section{L'INTRODUCTION}

\section{DE LA MÉDICATION}

Si les situations de risques suicidaires sont de puissants incitatifs à la consultation médicale, elles mènent aussi à la prescription de médicaments. En effet, à la salle d'urgence, une médication psychotrope est généralement introduite, surtout lorsqu'il n'y a pas de risque suicidaire imminent qui nécessiterait une hospitalisation. Ce médicament, généralement un antidépresseur, vient alors signer de manière concrète et tangible qu'une intervention d'urgence, se voulant plus incisive et plus percutante, a été effectuée. Dès lors, tous semblent considérer que le danger est moindre, qu'on a fait le nécessaire et que le jeune est désormais mieux "contrôlé ». Presque tous les éducateurs croient d'ailleurs que les pédopsychiatres prescrivent plus de médicaments psychotropes qu'auparavant.

- La pédopsychiatrie a un volet «prise de médicaments » presque assuré, là.

- Souvent ils vont ressortir de là avec une prescription, si on voit que le risque n'est pas immédiat, exemple: c'est un cas suicidaire. [...] Souvent, ils vont prescrire ça à l'urgence, puis après ils disent: "Tu prends rendezvous au service des enfants. Puis là, ça sera pour savoir si elle sera rencontrée. Puis pour savoir si on continue la médication. »

Trois cas de figure dépeignent un peu plus précisément l'introduction de la pharmacothérapie chez les jeunes placés. La médication peut d'abord être introduite antérieurement au placement.

- Souvent quand des jeunes nous arrivent, elles prennent déjà des médicaments. J'en avais une, un moment donné. Elle s'était jetée en bas d'un deuxième étage. Il y avait eu une tentative de suicide grave et importante. Elle avait douze ans et quand elle nous est arrivée, elle était déjà médiquée [sic] de Sainte-Justine en pédopsychiatrie. Ce que je veux dire c'est que souvent, ce n'est pas dans une situation...

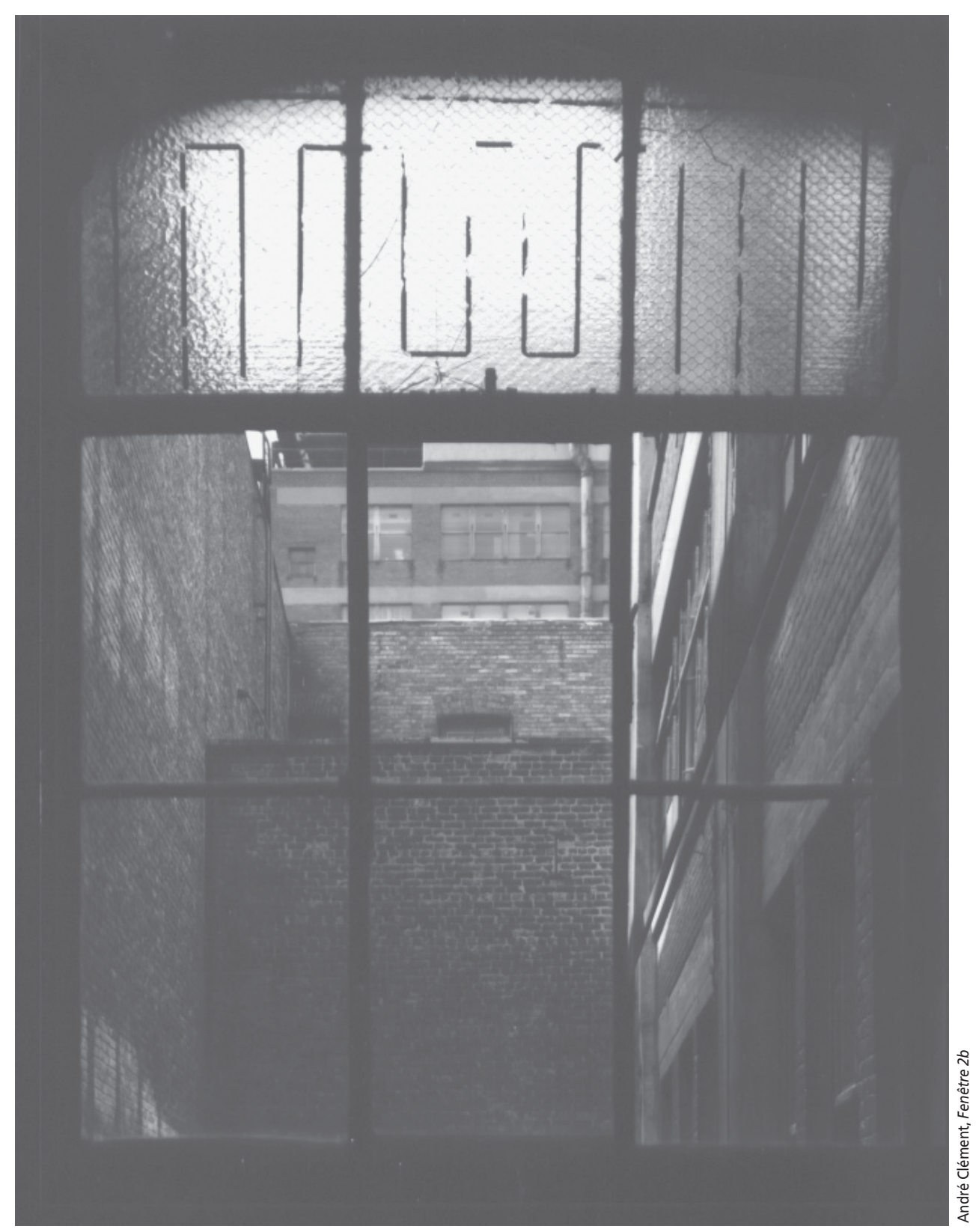

Cette conjoncture pose certaines difficultés pour les éducateurs, qui ne savent pas précisément pourquoi la pharmacothérapie a été introduite. Qui plus est, le psychiatre actuel n'est pas nécessairement celui qui a introduit la médication. Dans ce contexte, l'intervenant ne peut habituellement comparer les symptômes et comportements du jeune à ceux qu'il présentait avant l'introduction de la molécule et il lui devient très difficile d'évaluer et de réévaluer l'efficacité et la pertinence du traitement.

La pharmacothérapie peut ensuite être offerte parce que l'équipe juge qu'il y a une détérioration progressive de l'état du jeune résidant. Ainsi, au fil des mois, les intervenants peuvent observer l'apparition ou l'exacerbation de comportements inquiétants. Il semble être ici question surtout de symptômes évoquant des troubles psychotiques, des comportements désorganisés ou désorientés, un manque de jugement et la prise de risques importants (p. ex. fugues nocturnes et dangereuses).

Selon un troisième et dernier scénario, le médicament est introduit à la suite d'une situation d'urgence, ce qui est généralement le cas lorsqu'il y a tentative de suicide ou verbalisation suicidaire. Dans les deux régions où évoluent les répondants, la problématique de suicide représente le plus important motif de consultation en pédopsychiatrie. En effet, les non-médecins semblent très attentifs, vigilants aux moindres propos laissant présager un risque suicidaire.

- C'est toujours pour des idéations suicidaires ou des tentatives de suicide. Il n'y a pas vraiment d'autre situation susceptible de conduire un jeune à l'urgence. 


\section{LE RAPPORT AMBIVALENT DES ÉDUCATEURS AUX MÉDICAMENTS PSYCHOTROPES}

La majorité des éducateurs estime que la pharmacothérapie est pertinente et efficace, du moins sous certaines conditions, c'est-à-dire pourvu que le jeune se sente impliqué dans un travail thérapeutique ou rééducatif et qu'il soit suivi et accompagné par les intervenants médecins ou nonmédecins. Le médicament psychotrope est alors perçu comme une mesure de stabilisation des troubles, qui vient apaiser une détresse trop intense. La position de ces non-médecins est que la molécule doit être prescrite de façon temporaire. Ils s'attendent à ce que les médicaments prescrits aident le jeune à considérer des situations problématiques ou très souffrantes avec plus de calme et de manière plus contenue. Ils aimeraient aussi que les médicaments viennent soutenir leur propre action éducative en rendant le jeune plus accessible.

Les risques d'intoxication par une accumulation clandestine de médicaments sont considérés minimes, compte tenu des mesures sécuritaires mises de l'avant. Ainsi, à l'intérieur de l'unité de vie ou du foyer de groupe, les adolescents n'ont jamais les médicaments à leur disposition. Ceux-ci sont plutôt gardés sous clé dans le bureau des éducateurs et administrés en main propre, un jeune à la fois. En outre, l'introduction des "dosettes », au cours des dernières années, est décrite comme une innovation qui facilite le contrôle de la circulation des capsules et comprimés. S'il subsiste des risques d'intoxication, ils sont attribués aux moments sans surveillance vécus lors de sorties provisoires (fins de semaines ou congés fériés).

L'usage de la pharmacothérapie ne fait toutefois pas l'unanimité chez les éducateurs en centres jeunesse. Cette pratique pose problème aux yeux d'une minorité d'intervenants qui hésitent à administrer des médicaments psychotropes. Leur perplexité et leurs réticences reposent parfois sur le sentiment d'un manque d'information sur les produits prescrits, les risques de surdose, la crainte que les jeunes fassent un usage hédoniste des médicaments ou l'inquiétude devant de possibles interactions entre ces molécules, de l'alcool ou des drogues de rue.

\section{- Qu'est-ce qui est embêtant, c'est de savoir s'ils sont toujours pris d'une manière, euh, comme prescrit avec les jeunes. Quels autres mélanges qu'ils peuvent faire?}

- Ça ne veut pas dire aussi que ton jeune va prendre sa médication. Hein, ça aussi, des fois, ils reviennent avec leurs enveloppes. «J'ai oublié. » Ça fait partie de la game, ça, je te dirais.
Hormis ces propos sur la méconnaissance ou les «détournements» du traitement, les réserves émises par d'autres interviewés témoignent d'une position plus sceptique et critique envers le traitement lui-même. La première de ces remises en question porte sur la discontinuité perçue des suivis médicaux. Pour certains, l'espacement des entretiens avec le médecin peut devenir si considérable (un bref rendezvous tous les ans) que le traitement psychotrope paraît perdre tout son sens et se dérouler dans un contexte discutable au plan déontologique.

- Il y en a qui y allaient aux mois. Puis, il y en a d'autres qui y allaient aux ans. Ils prescrivaient pour un an et tu $\mathrm{y}$ retournais au bout d'un an, à moins qu'il y ait des événements particuliers. Ça pouvait être de l'automutilation assez importante, des verbalisations suicidaires, des tentatives suicidaires, une désorganisation avec une perte de contrôle, de l'agressivité, euh... Ça prenait vraiment des événements qui sortaient de l'ordinaire pour avoir un rendez-vous.

- Tsé, elle allait avoir 18 ans dans une couple de semaines... Puis elle a demandé à son psychiatre au téléphone: "Je me sens plus nerveuse. Est-ce que tu voudrais augmenter ma médication? » Et ça s'est fait. Moi, je trouve que c'est des situations qui n'ont pas de sens au niveau clinique, que tu puisses comme ça, par téléphone, renouveler une prescription ou faire augmenter une dose sans que le médecin ait vu la jeune adolescente. Ça s'est fait.

L'autre source de questionnement tient au fait que la médication, qui paraît si régulièrement prescrite, semble avoir l'effet pervers de désamorcer soudainement l'urgence perçue, tout en démobilisant l'ensemble des proches et des soignants. En d'autres termes, ces interviewés jugent que la pharmacothérapie devient un expédient commode, rapide, qui finit par retarder ou occulter la mise en place d'autres interventions.

- Il part, mais la résidente dit: «Bon, on va prendre un rendez-vous, normalement, avec un psychiatre de l'équipe du Centre des adolescents. » Ce n'est pas la semaine prochaine, hein, c'est dans trois mois. On reçoit un appel un moment donné. Une travailleuse sociale de l'équipe qui nous demande: «Ben, le jeune comment ça va? Il n'est pas très urgent hein? S'il est chez vous et tout ça. » Nous on dit: «Mais madame, on a besoin d'un rendezvous. » Elle répond: «Oui, mais là ça va bien. Il est médicamenté votre jeune. Finalement, là ça va. »

- Ça se peut qu'ils me portent très peu d'intérêt parce qu'ils savent très bien que c'est le protocole qui fait qu'on n'a pas le choix de consulter. [...] Souvent ils vont faire une évaluation très sommaire. «Il n'est pas en danger? Bien. À c't'heure, prends la bonne porte, comprends-tu?»

\section{PROTOCOLES OU STABILITÉ ET CONTINUITÉ DES LIENS ?}

Autopsie: «Examen d'un cadavre pour déterminer les causes de la mort» (Petit Larousse, 2002. p. 102). Enquête: «Ensemble de recherches ordonnées par une autorité administrative ou judiciaire » (Petit Larousse, 2002, p. 383).

Tout suicide d'enfant ou d'adolescent est un drame qu'il faut tenter de prévenir, personne ne voudra le nier. Cela dit, comment réagir lorsque survient l'horreur? Entreprendre une recherche, une autopsie ou une enquête? Si tous les termes évoquent le désir de comprendre, il est difficile de ne pas signaler que le second (autopsie) renvoie à l'expertise médicale, tandis que le dernier (enquête) évoque la culpabilité et la responsabilité.

Par rapport aux événements que nous avons relatés, deux éléments peuvent surprendre. Il y a tout d'abord cette décision du coroner David de confier initialement l'étude des causes de suicides antérieurs au seul corps médical ( un comité de pédopsychiatres »). Il y a lieu de se demander si les médecins seraient les seuls à pouvoir procéder à une "autopsie », lorsque cette recherche devient à la fois biologique, psychologique et sociale? Faut-il comprendre que cette quête de sens " après coup » interpelle tous les intervenants, mais qu'elle est la prérogative du médecin?

Vient ensuite cette vérité voulant que la condition suicidaire est « dans $85 \%$ des cas » sous-tendue par un processus morbide, relevant de la maladie mentale. Quelques passages du rapport de 2001 mentionnent bel et bien que d'autres facteurs, tels que l'impulsivité, la toxicomanie, les pertes relationnelles, les conflits interpersonnels et les changements de milieux subis par la clientèle, peuvent intervenir dans le passage à l'acte suicidaire. Aucun modèle intégratif n'est toutefois proposé pour rendre plus cohérente cette coexistence d'une terminologie médicale et d'une terminologie plus propre à la psychoéducation. Dans un travail antérieur, nous avons signalé que le point de fragilité le plus profond et complexe dans la relation médecin / non-médecin relève sans doute 
des modèles théoriques (Lafortune, 1998). En effet, des tensions conceptuelles risquent de s'inscrire autour des clivages corps / esprit, démarche scientifique / démarche humaniste, traitement relationnel dit « naturel » / traitement pharmacologique dit « chimique». La formation médicale peut s'appuyer parfois sur un organicisme strict, selon lequel «les maladies mentales sont des maladies $d u$ cerveau ». De la même façon, les formations humaniste, cognitivo-behaviorale ou psychanalytique, peuvent avoir du mal à prendre en compte réellement la dimension organique, les neurotransmetteurs, les synapses et les structures anatomiques. Les auteurs du rapport sur le suicide déplorent les chasses gardées et les cloisonnements disciplinaires mais ils n'offrent aucune issue au plan conceptuel, si ce n'est un scénario où tous se rallieraient au modèle bio-psychosocial. Or il est difficile d'oublier que ce modèle provient de la psychiatrie, au même titre que le DSM-IV. Faut-il ici comprendre qu'aux yeux du Comité, le modèle intégrateur et rassembleur des diverses professions devrait être le modèle médical ?

À ces deux premières questions, nous ajouterons deux remarques, l'une sur la notion de partenariat et l'autre sur la décision clinique. Les principales conclusions $\mathrm{du}$ rapport suggèrent que si quelque chose a manqué dans la prise en charge des jeunes décédés, ce doit être le nombre de médecins, l'accès aux services qu'offrent ces médecins ou encore la concertation entre les milieux médicaux et les centres jeunesse. Les solutions proposées gravitent alors autour de la formation continue, des protocoles conjoints et des procédures à appliquer systématiquement. Tous se rallieront aisément à cette proposition: la leçon à tirer des drames du passé est qu'il vaut mieux maintenir des partenariats stratégiques, advenant qu'une situation urgente se présente. Or, le point faible de cette entreprise nous semble être la mobilité du personnel dans les deux milieux concernés. On dit que les organismes doivent travailler en partenariat mais, de fait, ce sont les personnes qui doivent le faire. Avec chaque nouvel intervenant ou résidant qui passe, il faut renouer les liens et redécouvrir les façons de faire de son collègue. La mobilité des soignants, l'instabilité des équipes et la discontinuité des liens nous paraissent être à la source d'importants hiatus.

Reste le contexte dans lequel se prennent maintenant les décisions cliniques. Le contenu des entretiens semi-structurés nous a appris que la majorité des éducateurs apprécie le nouveau protocole d'intervention, ainsi que cette philosophie qui promeut la concertation, la collaboration entre les intervenants et le partage des responsabilités. Néanmoins, le contexte « d'enquête » en a rendu plus d'un craintif. Il faut s'interroger sur les effets qu'ont eu ces événements, osons dire "traumatiques", sur l'ensemble des éducateurs. Ont-ils installé un doute nécessaire et salutaire invitant à l'auto-évaluation des pratiques? Dans le sens où «la crainte est le début de la sagesse »? Ont-ils plutôt instauré la mise en place de réflexes et d'automatismes visant surtout à "se couvrir» face à de possibles blâmes?

Devant le risque suicidaire, une expérience des plus courantes est celle de «faire trop peu». Une autre tourne autour du temps et de l'impression que le processus est lent ou qu'il piétine. L'un des répondants disait d'ailleurs ne "pas être équipé » pour intervenir sur ces questions. En adressant le jeune vers un confrère médical, il semble s'agir, pour certains éducateurs, de se dégager d'un sentiment d'insécurité, d'impuissance et «d'insuffisance ». Se sentant démuni ou inquiet, l'éducateur envisage parfois la pharmacoprescription comme une intervention plus active, incisive ou percutante, "provoquant quelque chose». Dans toute pratique clinique avec les mineurs, la détresse et l'immaturité des jeunes sont les meilleurs incitatifs possibles à l'activité ou à l'activisme des intervenants. Il en va de même de la présence des parents, des collègues et du Tribunal qui, de près ou de loin, observent l'évolution de l'enfant. À cette pression s'est ajoutée depuis neuf ans une campagne de sensibilisation... accompagnée de plaintes, d'enquêtes et d'autopsies psychologiques. Le regard des collègues et supérieurs paraît exercer maintenant une pression et, pour certains, susciter de l'inquiétude et la crainte du blâme. En de tels cas, la distinction n'est pas toujours facile à faire entre une décision éclairée, réfléchie et un passage à l'acte contretransférentiel. N'est-il pas « urgent d'agir », selon le comité triparti ? Il reste donc à voir si la tension ambiante sera féconde ou paralysante.

Pour conclure, il est frappant de constater combien est maintenant admise cette idée voulant $a$ ) qu'en centres jeunesse l'évaluation des risques suicidaires relève de tous les intervenants, mais que l'expertise et l'intervention d'urgence sur ces mêmes risques appartiennent en priorité au pédopsychiatre, $b$ ) parce qu'une maladie mentale (probablement la dépression) est en cause et que $c$ ) des médicaments psychotropes pourraient être prescrits. Ce discours paraît faire l'objet d'un tel consensus, être présenté comme une telle vérité que l'on se demande bien qui, parmi les praticiens, pourrait soutenir un autre point de vue sans passer pour un irresponsable rétrograde qui joue avec la vie des jeunes et leur droit à des services de qualité.

\section{Bibliographie}

BOUCHARD, R. (2002). Psychopharmacologie et prise en charge des adolescents dans les Centres jeunesse, mémoire de maîtrise inédit, École de criminologie, Université de Montréal.

BUREAU DU CORONER (1998). Rapport annuel 1998; Dossier: A-121592 94522, Sainte-Foy, Les Publications du Québec.

BOUSQUET, J. (2002). "Suicide, plus qu'un mal de vivre », Art de vivre; Société, Document en ligne $<$ http://www.canoe.qc. ca/artdevivresociete/mar19_02_maldevivre _ c-can.html $>$, consulté le 5 juin 2003.

COLLÈGE DES MÉDECINS DU QUÉBEC (2001). Lauréats du Grand Prix $2001 d u$ Collège des médecins du Québec, Document en ligne: <http://www.cmq.org/pages/ sections/cenmedia/communiq/20010511. html>, consulté le 5 juin 2003.

COMITÉ SUR LE SUICIDE CHEZ LES JEUNES (1999). «Le suicide chez les usagers des centres jeunesse : il est urgent d'agir!», Québec: Le Protecteur du citoyen, Assemblée nationale.

LAFORTUNE, D. (1998). «Entre le remède et la relation, le regard d'un non-médecin ", P.R.I.S.M.E., vol. 8, $\mathrm{n}^{\circ}$ 1, p. 50-69.

LAFORTUNE, D. et C. LAURIER (2003) Les drogues médicales... ou le recours aux médicaments psychotropes dans la prise en charge des jeunes hébergés dans les centres jeunesse, Soumis pour publication.

LAFORTUNE, D., C. LAURIER et F. GAGNON (2003). Prévalence et facteurs associés à la prescription de médicaments psychotropes chez les sujets placés en centre jeunesse, Soumis pour publication.

PRONOVOST, J. et D. LECLERC (1998). Le dépistage des adolescent(e)s suicidaires en centres jeunesse. Rapport final: faits saillants, Université du Québec à Trois Rivières. 